\title{
KAWASAKI DISEASE - A CASE REPORT
}

\section{Shrestha S*, Adhikari N}

\section{INTRODUCTION}

Kawasaki disease, first described by Tomisaku Kawasaki in 1967 , is an acute systemic vasculitis of infancy and childhood. It is now the leading cause of acquired heart disease in developed countries. The most common age group affected is between 6 months to 5 years and the peak incidence is in children aged 9 to 11 months. ${ }^{1}$ The disease has male preponderance with the male female ratio of $1.5: 1 .^{2}$ Though the exact etiology is not known the disease is known to be associated with unusual degree of immune activation and immunoregulatory abnormality. ${ }^{3}$

\section{CASE REPORT}

A one and a half year old boy presented to us with the history of high-grade continuous fever for 4 days, skin rash mainly over face and trunk for 3 days, irritability, poor feeding and loose stool with mucus for 2 days. Before coming to us the boy was seen by a doctor and was given amoxycillin but after 2 doses of the drug, parents noticed skin rash and antibiotic was then changed to cefaclor. The child had 2 days of cefaclor from outside before seeing us.

On examination, he was febrile, very irritable, toxic and sick looking. There was macular rash over the face neck, trunk and arms. There was bilateral mild conjuctival congestion.

He had hyperaemic pharyngeal and oral mucosa. There were few palpable, non tender cervical nodes measuring 1.5 to $2 \mathrm{~cm}$ in size and one tender left submandibular node of $3 \times 3 \mathrm{~cm}$ size.

* Patan Hospital, Patan, Nepal. Address for correspondence :
Dr. Shrijana Shrestha

Patan Hospital

GPO Box: 252, Kathmandu, Nepal

Email: patan@hospital.wlink.com.np
The boy was admitted with the provisional diagnosis of viral exanthema with viral meningitis and the differential diagnoses then were: 1 . Partially treated bacterial meningitis with drug rash: 2. Staphylococcal bacteraemia / sepsis with lymphadenitis .As the child was sick, was started empirically on injection ceftrioxone $(100 \mathrm{mg} / \mathrm{kg})$ and injection cloxacillin $(100 \mathrm{mg} /$ $\mathrm{kg}$ ) after sending samples of cerebrospinal fluid (CSF), blood and urine for cultures.

The next day a few new findings were noted. There were cracked lips with red strawberry tongue. The skin rash became more blotchy and diffuse extending to the thighs.

Swelling of the extremities involving the hands and legs with non-pitting edema was noted.

The investigation reports showed peripheral white blood cell count (WBC) of $14,200 / \mathrm{mm}^{3}$ with neutrophil $57 \%$ and lymphocyte $43 \%$. The CSF analysis showed WBC of 9, neutrophil 3, lymphocyte 6 , protein 32 and sugar $70 \mathrm{mg} / \mathrm{dl}$. Urine examination showed trace albumin with 2-3 WBC. Stool examination showed 20-22 WBC and 0-2 RBC (Red blood cell). Erythrocyte sedimentation rate (ESR) was $78 \mathrm{~mm} /$ hour. Platelets count, chest $\mathrm{x}$ - ray and electrocardiography were normal.

High-grade fever continued in the hospital and persisted beyond 48 hours of intravenous antibiotics (ceftrioxone and cloxacillin). At 48 hours the cultures of CSF, urine, stool and blood all were negative.

With these findings, clinical course and investigations the diagnosis of Kawasaki disease was reached as the clinical criteria 
for diagnosis was fulfilled. The supportive investigation results were leucocytosis, CSF pleocytosis with normal protein and sugar, and elevated ESR. On the third admission day, that is the $7^{\text {th }}$ day of illness intravenous immunoglobulin (IVIG) $2 \mathrm{gm} / \mathrm{kg}$ single dose was infused and aspirin in a dose of $100 \mathrm{mg} / \mathrm{kg}$ was started.

After 24 hours of immunoglobulin infusion, fever resolved and rash disappeared. Irritability and limb swelling started to decrease and resolved after 48 hours of the treatment.

The final blood culture report was negative after 7 days of inoculation. On the $7^{\text {th }}$ hospital day the boy was discharged on oral aspirin and called for follow up after 5 days with the report of Echocardiogram.

During the follow up visit, on the third week of illness, skin peeling of his hands and feet were noted which had started on $15^{\text {th }}$ day of the illness.

Thrombocytosis with the platelet counts of $428,000 / \mathrm{mm}^{3}$ was noted and ESR had come down to $37 \mathrm{~mm} /$ hour. The echocardiography was reported normal with no evidence of proximal coronary artery aneurysm. The dose of aspirin was then decreased to $5 \mathrm{mg} / \mathrm{kg}$ and was advised for a follow up echocardiography at 8 weeks of illness.

\section{DISCUSSION}

Kawasaki disease is the second commonest vasculitis syndrome of childhood. It is a multisystemic vasculitic disease affecting medium sized arteries and is complicated by the development of coronary and peripheral arterial aneurysm ${ }^{4}$. Coronary artery aneurysm that is the most dreaded complication of Kawasaki disease occurs in 10 to $40 \%$ of untreated cases. Almost about $50 \%$ of the aneurysms resolve within five years. Giant aneurysms $(>8 \mathrm{~mm})$ are unlikely to resolve and is complicated by coronary thrombosis, stenosis and myocardial infarction. The diagnosis

\section{Table I: Clinical diagnostic criteria for Kawasaki disease ${ }^{4}$}

1. Fever for at least 5 days

2. Presence of 4 out of the following 5 conditions

a. B/L non purulent conjunctivitis

b. Changes in the mucosa of the oro pharynx, fissured lips, strawberry tongue

c. Changes in the peripheral extremities: edema, erythema \& desquamation

d. Rash primarily truncal, polymorphous, non vesicular

e. Cervical lymph adenopathy

3. Illness not explained by other known disease processes is mainly clinical and no single laboratory test helps to confirm the diagnosis. In this childhood vasculitis, constellation of clinical findings appear sequentially over days to weeks. None of the clinical features taken individually are of any significance.

On the basis of this diagnostic criteria cases are classified as complete case that fulfils the diagnostic criteria and incomplete case, which has fewer than the requisite criteria. Though coronary artery aneurysm is not included in the diagnostic criteria, if present, requires only 3 of the other criteria with fever to make a complete case. An important point not to forget is that an incomplete case can evolve with time into a complete case. ${ }^{4}$

Some of the less specific but relatively common features of Kawasaki disease are: arthritis/arthalgia, aseptic meningitis, hepatic dysfunction, gastroenteritis, pneumonia, nephritis and otitis. Among the uncommon features of the disease are: jaundice, myocarditis, pericarditis, hydropes of gall bladder, seizures and ataxia.

The disease is associated with many non-specific laboratory findings and those are elevated acute phase reactants, leucocytosis, thombocytosis (towards the end of second week), CSF pleocytosis, elevated liver enzymes and sterile pyuria. ${ }^{5}$ Chest $\mathrm{x}$-ray electrocardiography and echocardiography are some important investigations for evaluating cardiac complications.

\section{Table II : Important signs, not included in the} diagnostic criteria ${ }^{4}$

\section{* Irritability:}

Almost universally present in cases of Kawasaki disease. The exact mechanism is not clear but may be related to the presence of aseptic meningitis.

* Erythema and induration at the site of $B C G$ vaccination:

This clinical sign is relatively specific to Kawasaki disease. The mechanism described is, cross reactivity of $\mathrm{T}$ cells in Kawasaki disease patients to specific epitopes of mycobacterial proteins. ${ }^{1,5}$

The differential diagnosis which needs to be considered and excluded are: toxic shock syndrome, Staphylococcal scalded skin syndrome, scarlet fever, viral infections like measles, EbsteinBar, some rickettsiael infections, Leptospirosis, polyarteritis nodosa and systemic onset juvenile rheumatoid arthritis. ${ }^{5}$

Our patient had fulfilled the clinical diagnostic criteria, fitting into the definition of a complete case. He also had some other important clinical feature like irritability, CSF pleocytosis and gastroenteritis. The patient showed dramatic response to 
treatment with IVIG as is expected in Kawasaki disease. Thombocytosis was observed in the third week of the illness.

The goal of treatment is directed towards reducing inflammation and preventing the development of coronary and peripheral arterial aneurysm. Aspirin given in high anti-inflammatory doses $(30-100 \mathrm{mg} / \mathrm{kg} /$ day) is useful for symptomatic relief. Recent reviews have recommended higher dose of $100 \mathrm{mg} / \mathrm{kg} / \mathrm{day}$ in acute phase of illness ${ }^{4}$ as it brings down the duration of fever and hospital stay. Following defervescence the dose is decreased down to $2-5 \mathrm{mg} / \mathrm{kg} /$ day for the anti platelate effect and is continued for 6 to 8 weeks. In case of presence of coronary artery aneurysm the low dose aspirin is continued for a longer time or till the aneurysm resolves.

Intravenous immunoglobulin (IVIG) is the only proven treatment to reduce the occurrence of coronary artery aneurysm provided that it is given early in the disease course that is within the first 7-10 days of illness. It can be used as a single dose infusion in a dose of $2 \mathrm{gm} / \mathrm{kg}$ or in divided doses of $400 \mathrm{mg} /$ $\mathrm{kg}$ /day for four consecutive days. Studies have shown better therapeutic benefit of single high dose infusion in terms of preventing coronary aneurysm formation. ${ }^{6}$ Single high dose infusions is also found to have more rapid defervesence. ${ }^{5}$ Some patients may require second infusion especially those who received IVIG very early in the disease course. IVIG can be given after the first 10 days of illness for symptomatic relief if signs of inflammation persist but its role in preventing coronary aneurysm is not proven. Given within the first 10 days of illness, IVIG decreases the incidence of coronary aneurysm down to $9 \%$ at 30 days and $4 \%$ at 60 days. $^{6}$

Unlike other vasculitic syndromes, the role of corticosteroids in Kawasaki disease is controversial some believe it is contraindicated as it might increase the incidence of coronary aneurysm. ${ }^{5}$ It might have some role in patients refractory to treatment with IVIG. ${ }^{7}$

There is on going research on Kawasaki disease being carried out by the UK Kawasaki research group. Some of the specific areas of research include Epidemiology, complication rate, case fatality rate; etiopathogenesis; host genetic determinants of susceptibility and outcome; correlation of aneurysm formation and clinical presentation; and development of a laboratory diagnostic test. ${ }^{4}$

\section{CONCLUSION}

Kawasaki disease is a self-limiting condition associated with abnormal immune response to a triggering factor, which probably is some infectious agent. It is difficult to establish the diagnosis of the disease in the initial phage of illness, yet it is crucial to make correct diagnosis early in the disease course in order to prevent serious complications and long term morbidity.

\section{REFERENCES}

1. Levin M, Tizard EJ, Dillon MJ. Kawasaki disease: recent advances. Arch Dis Child 1991; 66: 1369-74.

2. Mitra S, Singh S, Grover A, Kunour L.A child with prolong pyrexia $\&$ peripheral desquamation: Is it Kawasaki disease. Ind Pediatr 2000; 37: 786-89.

3. Sign S \& Das R. Clinical approach to vasculitides. Ind J Peds 2002; 69: 881-88.

4. Brogan PA, Bose A, Burgner D, Shingadia, et. al. Kawasaki disease: an evidence based approach to diagnosis, treatment and proposals for future research. Arch Dis Child 2002; 86: 286-90.

5. Shulman ST, Inocencio J, Hirsch R. Kawasaki disease. Pediatr Clin North Am 1995; 42: 1205-22.

6. Durongpisitkul K, Gururaj VJ, Park JM, Martin CF.The prevention of coronary artery aneurysm in Kawasaki disease: a meta analysis on the efficacy of aspirin and immunoglobulin trearment. Pediatrics 1995; 96: 1057-61.

7. Wright DA, Newburger JW, Baker A, Sundel RP. Treatment of immunoglobulin-resistant Kawasaki disease with pulsed doses of corticosteroids. J Pediatr 1996; 128: 146-49.

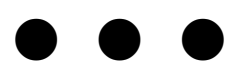

